\title{
Preparation and microstructure of cobalt(III) poly (acrylate) hybrid materials
}

\author{
Carlos Alvarez-Gayosso ${ }^{1}$, Miguel A. Canseco ${ }^{2}$, Rodolfo Estrada ${ }^{3}$, Joaquin Palacios-Alquisira ${ }^{4}$, \\ Jaime Hinojosa ${ }^{5}$, Víctor M. Castaño ${ }^{6} *$ \\ ${ }^{I}$ División de Estudios de Posgrado, Facultad de Odontología, UNAM \\ ${ }^{2}$ Instituto de Investigaciones en Materiales, UNAM \\ ${ }^{3}$ Universidad Iberoamericana \\ ${ }^{4}$ Facultad de Química, UNAM \\ ${ }_{5}^{5}$ Facultad de Estudios Superiores Cuautitlán, UNAM \\ ${ }^{6}$ Centro de Física Aplicada y Tecnología Avanzada, UNAM \\ *Corresponding author E-mail: meneses@unam.mx
}

Copyright (1) 2015 Víctor M. Castaño et al. This is an open access article distributed under the Creative Commons Attribution License, which permits unrestricted use, distribution, and reproduction in any medium, provided the original work is properly cited.

\begin{abstract}
The chemical reactions between poly (acrylic acid) and cobalt (III) oxide and between poly (acrylic acid) and cobalt (III) fluoride were studied by Fourier Transform Infrared Spectroscopy. The results demonstrate the formation of cements between the metal and PAA aqueous solutions. Structural information was obtained from the observed frequency shifts of the absorption bands of the carboxylates groups in the cobalt (III) poly (acrylate) relative to those recorded for the purely ionic form. The results show that the bidentate bridging structure is the most likely to occur in both cases. Also, from the analysis of the corresponding coordination chemistry, an octahedral structure may be tentatively assigned to the cobalt (III) poly (acrylate).
\end{abstract}

Keywords: Polyelectrolyte Compounds; Hybrid Materials; Polyacrylic Acid; Cobalt Compounds; Nanocomposites.

\section{Introduction}

Poly(acrylic acid) (PAA) and its salts have been known to have useful binding properties for many years. This salts, and poly(acrylic acid) have been used for soil consolidation and as flocculating agents. When poly(acrylic acid) is used for soil consolidation, one is basically just employing one among the vast range of acid-base phenomena, which occur throughout both inorganic and organic materials. Just like Portland cement, the above materials are prepared by mixing a powder with a liquid. However, in the case of PAA-based cements, this liquid phase is not water but an acid, while the powder, a metal oxide, is a base. Not surprisingly, the cement-forming reaction between them is extremely rapid and a hardened mass is formed within minutes of mixing. The product of the reaction, the binding agent, is a complex salt. Each cement system is a particular combination of an acid and a base. Cement-forming liquids are strongly hydrogenbonded and viscous. They must have sufficient acidity as to decompose the basic powder and liberate cement-forming cations, and they must act as a medium for the reaction. Generally, cement-forming liquids are aqueous solutions of inorganic or organic acids. These acids could be phosphoric acid, carboxylic acids and others. The bases are oxides and hydroxides of di and trivalent metals, some glasses, etc.

The setting reaction for the great majority of acid-base cements take place in water. Water thus acts both as a solvent and as a component in the formation of these cements. Also, it is one of the reaction products, being formed in the neutralization reaction as the cements set.

This kind of cements are not only formulated from relatively small ions with well defined hydration numbers. They may also be prepared from macromolecules, which dissolve in water to give multiply charged species known as polyelectrolytes. Cements which fall into this category are polycarboxylates. For example, the zinc (II) poly(acrylate) is formed when the neutralization reaction of zinc (II) oxide takes place in an aqueous solution of poly (acrylic acid). 
Polyelectrolytes form the basis of those cements, which are distinguished by their ability to adhere to reactive surfaces. Nowadays, the main use of such cements lies in the medical field, principally in dental surgery. They adhere permanently to biological surfaces where they must withstand adverse conditions of wetness, chemical attack, the stress of biological activity and chemical and biological changes within the substrate. Nevertheless, adhesive bonds are maintained.

Polyelectrolytes are polymers having a multiplicity of ionizable groups. In solution, they dissociate into polyions and small ions of the opposite charge, known as counterions. One typical polyelectrolyte is the poly(acrylic acid). The shape or configuration of a polyion is known as its conformation. There are many possible conformations available to a polyion because of the flexibility of the main chain due to the free rotation of bonds. There are two broad kinds of polyion conformation: the random coil and the ordered helix. Random coil conformations can range from spherical contracted state to the fully extended cylindrical or rod-like form. The conformation adopted depends on the charge on the polyion and the effect of the counterions. When the charge is low the conformation is a contracted random coil. As the charge increases the chain extends under the influence of mutually repulsive forces to a rod-like form. Thus, when a weak polyelectrolyte acid is neutralized, its conformation changes from a compact random coil to an extended chain. Poly(acrylic acid) shows this behaviour [8].

As mentioned before poly(acrylic acid) and its salts have been known to have useful binding properties for some years; they have used for soil consolidation [1], [2], [11-13,[16], [19], [22], [23], [25], [27-32] and as flocculant [24].

The most common poly(alkenoic acid) used in polycarboxylate cements is poly(acrylic acid), PAA. These poly(acids) are prepared by free-radical polymerization in aqueous solution using ammonium persulfate as the initiator and isopropyl alcohol as the chain transfer agent [5]. Under these conditions, the molecular weight of the PAA obtained lies below 10,000 .

The cement-forming reaction of the polyelectrolyte cements may be considered to take place in a number of overlapping stages. These are the attack by the acid on the base, the migration of the liberated ions from the base in the aqueous phase, the ionization of the poly(acid) with consequent unwinding of the polymer chain, the interaction between the charged chains and cations leading to ion binding and gelation, and the hardening phase [3].

The molecular structure of the polyelectrolyte cements has been examined by a number of workers [19], [22], [29], [30], [32] using infrared spectroscopy. Empirical correlations between the carbon-oxygen stretching frequencies of the carboxylate ions and the nature of their coordination have been used [9], [20]. They basically consider the difference between the asymmetric and symmetric stretching frequencies, of the carboxylate group, to discern among different possible structures. A carboxylate ion can coordinate to metals in three ways: monodentate ligand, bidentate chelating ligand and bidentate bridging:

Ionic metal carboxylates are also possible, but in these compounds the cations are constrained to remain in the vecinity of the carboxylates anion by a general electrostatic field and there are no specific binding sites. Sodium poly(acrylate) is an example of ionic carboxylate.

Infrared spectroscopy offers a useful means of studying interactions betwen cations and carboxylate groups in liquids, solids and gels. Vibrations due to the carboxylic acid, $-\mathrm{COOH},\left(\sim 1700 \mathrm{~cm}^{-1}\right)$ can be distinguished from those of the carboxylates ion, $-\mathrm{COO}^{-1}$, and are affected by hydrogen bonding. The $\mathrm{C}-\mathrm{O}$ stretching vibrations of the carboxylate ion are influenced by the action. The asymetric mode is more sensitive than symetric mode. These frequencies vary from 1650 to $1500 \mathrm{~cm}^{-1}$.

The goal of this study is to show the reaction of metal oxide and metal halide with poly(acrylic acid) by infrared spectroscopy. Also we propose a possible structure of cobaltic salt of the PAA, using empirical correlations between the carbon-oxygen stretching frequencies of the carboxylate ions and the nature of their coordination.

\section{Experimental procedure}

\subsection{Materials}

Solutions of aqueous poly(acrylic acid), $30 \% \mathrm{w} / \mathrm{w}$, were prepared by polymerizing acrylic acid using the procedure of solution polymerization [5]. The PAA $(8,000 \mathrm{~g} / \mathrm{mol}$ average viscosimetric molecular weight) was prepared using as initiator ammonium persulfate and a chain-transfer agent 2-propanol. The initiation was carried out by means of free radicals who were obtained from the descomposition of ammonium persulfate. The dispersion was thermodynamically unstable and needed continuous agitation to prevent the coalescence and to dissipate the reaction heat. The polymerization required nitrogen to displace the oxygen which acts as inhibitor of the reaction. Polymerization was carried at $701{ }^{\circ} \mathrm{C}$ in a stainless steel Parr Reactor of $300 \mathrm{~mL}$ with stirrer, control temperature and with nitrogen purge. The identification of the PAA was made by infrared spectroscopy. The polymers were neither fractioned nor purified. Commercial Cobalt(III) Oxide $\left(\mathrm{Co}_{2} \mathrm{O}_{3}\right)$ was employed in this study. This is a black power $\left(95 \% \mathrm{Co}_{2} \mathrm{O}_{3}\right.$ and $\left.5 \% \mathrm{CoO}\right)$ from Union Miniere of Belgium. $\mathrm{Co}_{3} \mathrm{O}_{4}$ is your real chemistry formula; your name is tricobalt tetraoxide and your molecular weight: $241 \mathrm{~g} / \mathrm{mol}$. 
Commercially available Cobalt Trifluoride $\left(\mathrm{CoF}_{3}\right)$ was employed in this study. This is a light-brown power from Aldrich Chem. Co. It discolors rapidly on exposure to moist air. Molecular weight: $116 \mathrm{~g} / \mathrm{mol}$.

\subsection{Sample preparation}

Cobalt (III) poly (acrylate) was prepared by mixing PAA solution and metal oxide power or metal halide power and manually stirring the mixture for one minute on a glass block using a dental spatula. Pastes were prepared at $18^{\circ} \mathrm{C}$. A viscous black paste was obtained with cobalt (III) oxide. A viscous light yelow paste was obtained from the reaction with cobalt trifluoride. The material was submitted for analysis to determine the microstructure. All pastes were transferred to an $\mathrm{AgCl}$ crystal which was placed in the spectrometer as quickly as possible. The infrared measurements were taken using a Nicolet 510P spectrometer with a Nicplan IR-Microscope. Spectra were recorded a different times with 32 scans.

The number of moles of acrylic acid monomer units in PAA ware calculated, molecular weight: $72 \mathrm{~g} / \mathrm{mol}$. Different number of moles of $\mathrm{Co}_{3} \mathrm{O}_{4}$ and $\mathrm{CoF}_{3}$ were used.

The characterization of cobalt (III) poly(acrylate) was performed by comparing the FTIR spectra of unreacted PAA with samples of polysalts of cobaltus prepared as was explained above. The spectra were recorded between 4,000 and $400 \mathrm{~cm}^{-1}$. Band assignments are based on the standard texts by Nakamoto [20], Nyquist [26], and Bellamy [21]. Bands of particular importance to the present study were the carbonyl $\mathrm{C}=\mathrm{O}$ stretching mode of the carboxylic acid group and the asymmetric and symmetric carboxylic $\mathrm{COO}^{-1}$ stretching modes of the carboxylate groups.

\section{Results and discussion}

\subsection{Preparation of samples using Co3O4}

The reaction between PAA and $\mathrm{Co}_{2} \mathrm{O}_{3}$ and $\mathrm{CoO}$, considering PAA as the number of moles of acrylic acid monomer units in the polymer which is $\left(-\mathrm{CH}_{2}=\mathrm{CH}(\mathrm{COOH})-\right)$, is:

$8 \mathrm{PAA}+\mathrm{Co}_{2} \mathrm{O}_{3}+\mathrm{CoO} \sim 2 \mathrm{CoPA}^{\text {III }}+\mathrm{CoPA}^{\text {II }}+4 \mathrm{H}_{2} \mathrm{O}$

In fact the reaction shows two consecutive reactions. One yields cobalt(II) poly(acrylate) and other yields cobalt(III) poly(acrylate):

$2 \mathrm{PAA}+\mathrm{CoO} \sim \mathrm{CoPA}^{\mathrm{II}}+\mathrm{H}_{2} \mathrm{O}$

$6 \mathrm{PAA}+\mathrm{Co}_{2} \mathrm{O}_{3} \sim 2 \mathrm{CoPA}^{\mathrm{III}}+3 \mathrm{H}_{2} \mathrm{O}$

Figure 1 shows the Fourier transform infrared spectra of unreacted PAA and cobalt (III) poly (acrylate) -COPA-. The spectrum of unreacted PAA displayed bands at $\sim 3400 \mathrm{~cm}^{-1}\left(\mathrm{H}_{2} \mathrm{O}\right.$, reaction is in aqueous solution), $\sim 1700 \mathrm{~cm}$ (characteristic stretching, $\mathrm{COO}^{-1}$, this is marked with number 1), $\sim 1450 \mathrm{~cm}^{-1}$ and $\sim 1400 \mathrm{~cm}^{-1}$ (scissors and bending vibrations of $\mathrm{CH}_{2}$ and $\mathrm{CHCO}$ ) and $\sim 1230 \mathrm{~cm}^{-1}$ (OH bending of neighboring carboxyl group). The spectra of COPA exhibited bands at $\sim 1550 \mathrm{~cm}^{-1}$ (asymmetric $\mathrm{COO}^{-1}$ stretching, this is marked with number 2 ; the peak at $60 \mathrm{~s}$ is more short that the peak at $3240 \mathrm{~s}$ ), $\sim 1400 \mathrm{~cm}^{-1}$ (symmetric $\mathrm{COO}^{-1}$ stretching, this is marked with number 2). The characteristic stretching band of the carbonyl group shifted from $\sim 1700 \mathrm{~cm}^{-1}$ to $\sim 1550 \mathrm{~cm}^{-1}$. The peaks in $\sim 1230 \mathrm{~cm}$ in the unreacted PAA decreased in both spectra. These data indicates that cobalt (III) poly (acrylate) was present in the material. 


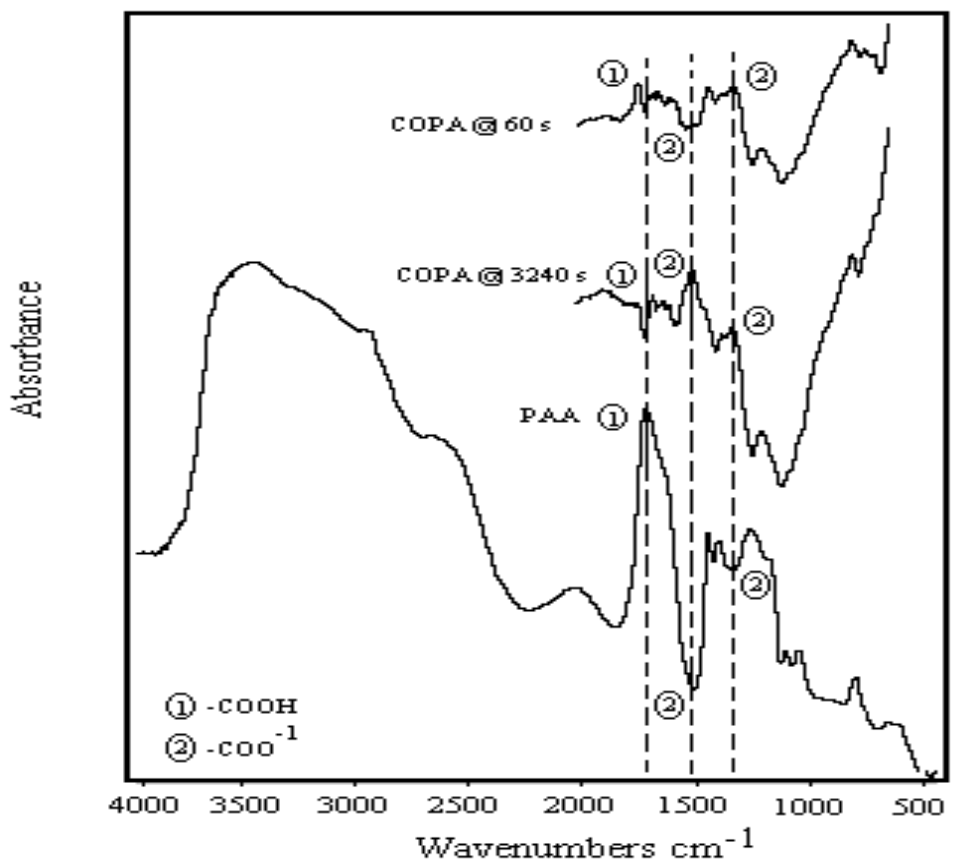

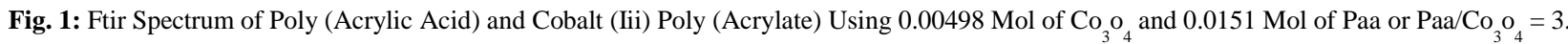

Figures 2, 3 and 4 show the FTIR spectra of unreacted PAA and cobalt (III) poly (acrylate) salts with different molar ratios of PAA and $\mathrm{Co}_{3} \mathrm{O}_{4}$.

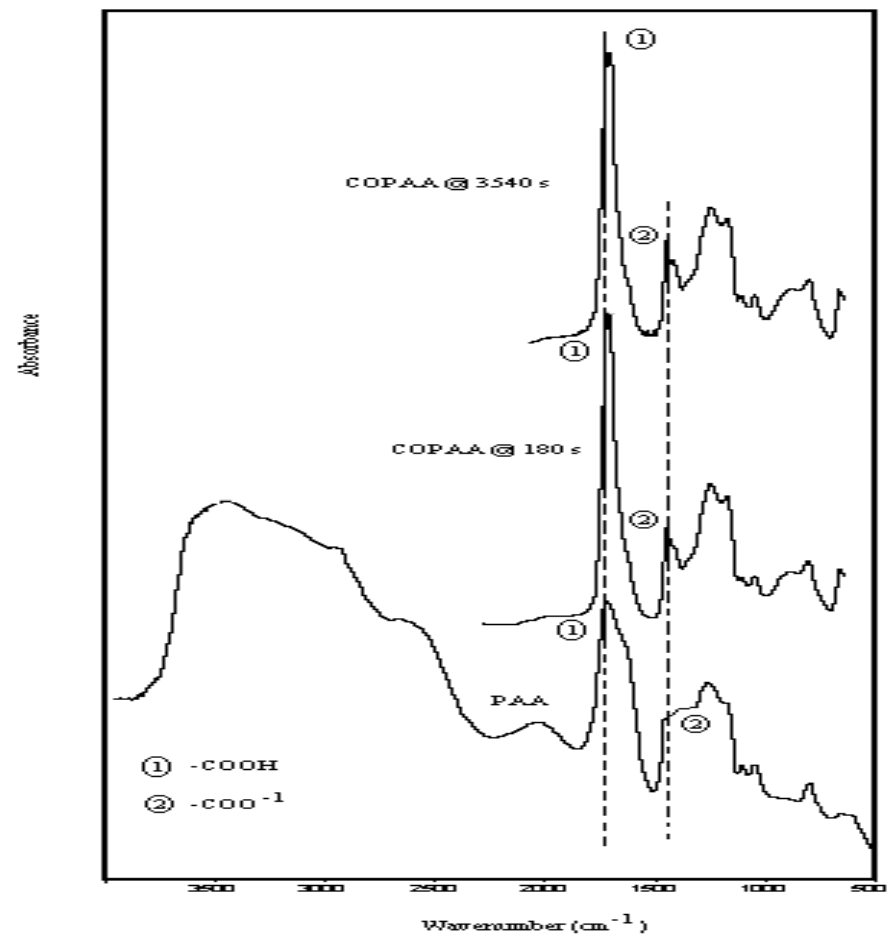

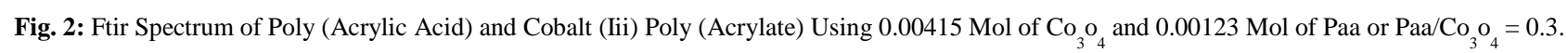




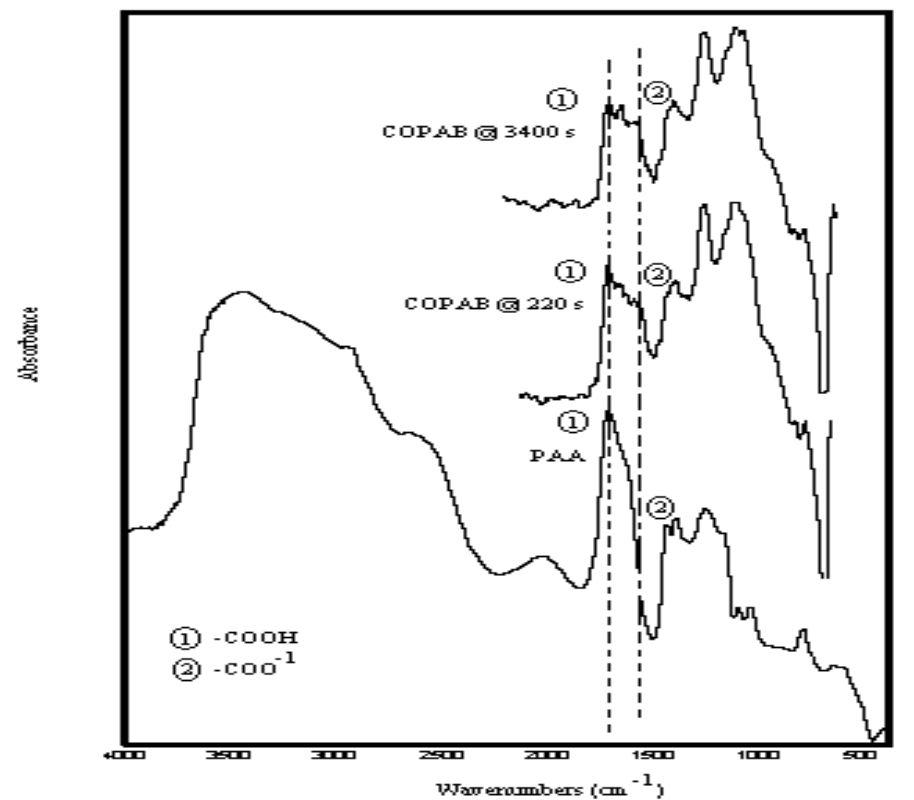

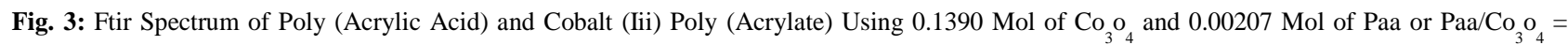
0.015 .

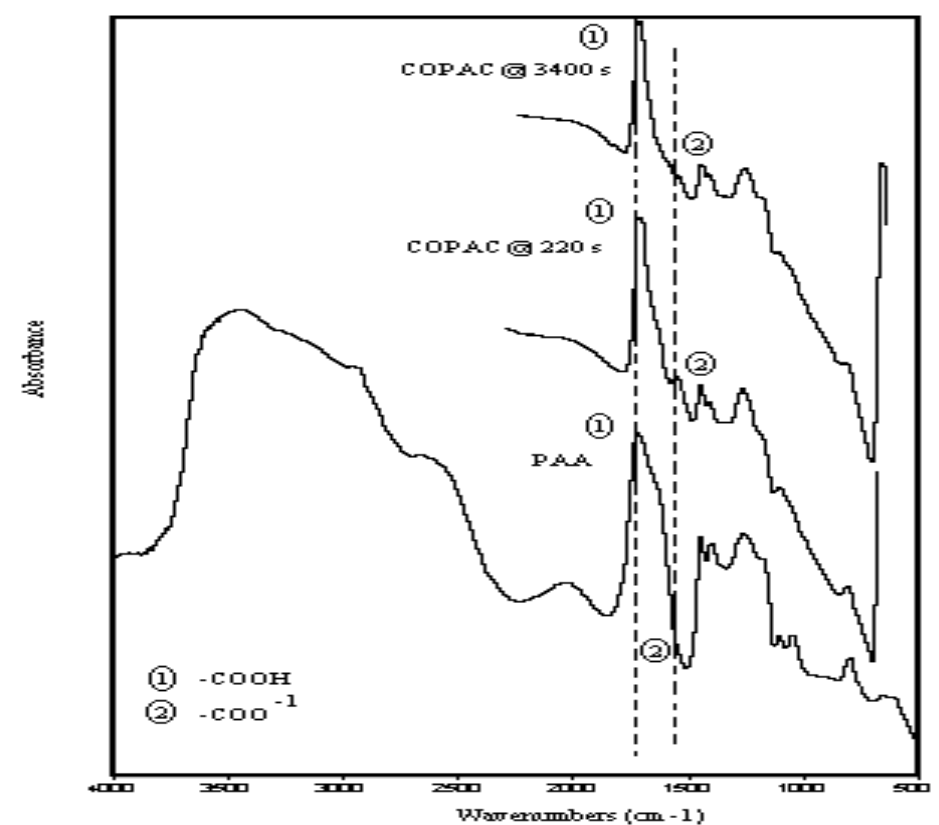

Fig. 4: Ftir Spectrum of Poly (Acrylic Acid) and Cobalt (lii) Poly (Acrylate) Using $0.3930 \mathrm{Mol}$ of $\mathrm{Co}_{3} \mathrm{o}_{4}$ and $0.01130 \mathrm{Mol}$ of $\mathrm{Paa}$ or $\mathrm{Paa} / \mathrm{Co}_{3} \mathrm{o}_{4}=$ 0.030 .

The general pattern of behavior observed in the reacting pastes of PAA with Co (III) compounds was for the $\mathrm{C}=\mathrm{O}$ stetching band (at $1700 \mathrm{~cm}$ ) of the carboxylic acid group to decline coincidently with the appearance and ehhancement of the $\mathrm{COO}^{-1}$ stretching bands of the metal carboxylates observed in the asymmetric stretch regions and the symmetric stretch regions. These results are indicative of an acid-base reaction.

\subsection{Preparation of samples using CoF3}

The reaction between PAA and $\mathrm{CoF}_{3}$, considering PAA as the number of moles of acrylic acid monomer units in the polymer which is $\left(-\mathrm{CH}_{2}=\mathrm{CH}(\mathrm{COOH})-\right)$, is:

$\mathrm{PAA}+\mathrm{CoF}_{3} \sim \mathrm{CoPA}^{\text {III }}+\mathrm{HF}$. 
As a matter of fact cobalt trifluoride reacts with water of PAA: [4], [14]

$\mathrm{CoF}_{3}+3 \mathrm{H}_{2} \mathrm{O} \sim \mathrm{Co}(\mathrm{OH})_{3}+\mathrm{HF}$

According with the process, the second reaction is:

$3 \mathrm{PAA}+\mathrm{Co}(\mathrm{OH})_{3} \sim \mathrm{CoPA}^{\mathrm{III}}+3 \mathrm{H}_{2} \mathrm{O}$

Figure 5 shows the Fourier transform infrared spectra of unreacted PAA, unreacted $\mathrm{CoF}_{3}$ and cobalt (III) poly(acrylate) -COPAD-. The spectrum of unreacted PAA displayed bands at $\sim 3400 \mathrm{~cm}^{-1}$ ( $\mathrm{H}_{2} \mathrm{O}$, reaction is in aqueous solution), $\sim 1700 \mathrm{~cm}^{-1}$ (characteristic stretching, $\mathrm{COO}^{-1}$, this is marked with number 1 ), $\sim 1450 \mathrm{~cm}^{-1}$ and $\sim 1400 \mathrm{~cm}^{-1}$ (scissors and bending vibrations of $\mathrm{CH}_{2}$ and $\mathrm{CHCO}$ ) and $\sim 1230 \mathrm{~cm}^{-1}$ (OH bending of neighboring carboxyl group). The spectrum of unreacted $\mathrm{CoF}_{3}$ displayed bands at $\sim 740 \mathrm{~cm}^{-1}$ and weak shoulder $\sim 860 \mathrm{~cm}^{-1}$ (this IR spectrum laboratory agrees with IR spectrum literature [26]). The spectra of COPAD exhibited bands at $\sim 1470 \mathrm{~cm}^{-1}$ (asymmetric COO ${ }^{-1}$ stretching, this is marked with number 2 ; the peak at $150 \mathrm{~s}$ is more short that the peak at $3905 \mathrm{~s}$ ), weak shoulder $\sim 1400 \mathrm{~cm}^{-1}$ (symmetric $\mathrm{COO}^{-1}$ stretching), the weak shoulder at $\sim 860 \mathrm{~cm}^{-1}$ became a real peak as the reaction continued. The characteristic stretching band of the carbonyl group shifted from $\sim 1700 \mathrm{~cm}^{-1}$ to $\sim 1470 \mathrm{~cm}^{-1}$. The peaks in $\sim 1230 \mathrm{~cm}^{-1}$ in the unreacted PAA decreased in both spectra. This occurrence indicates that cobalt (III) poly (acrylate) was present in the material.

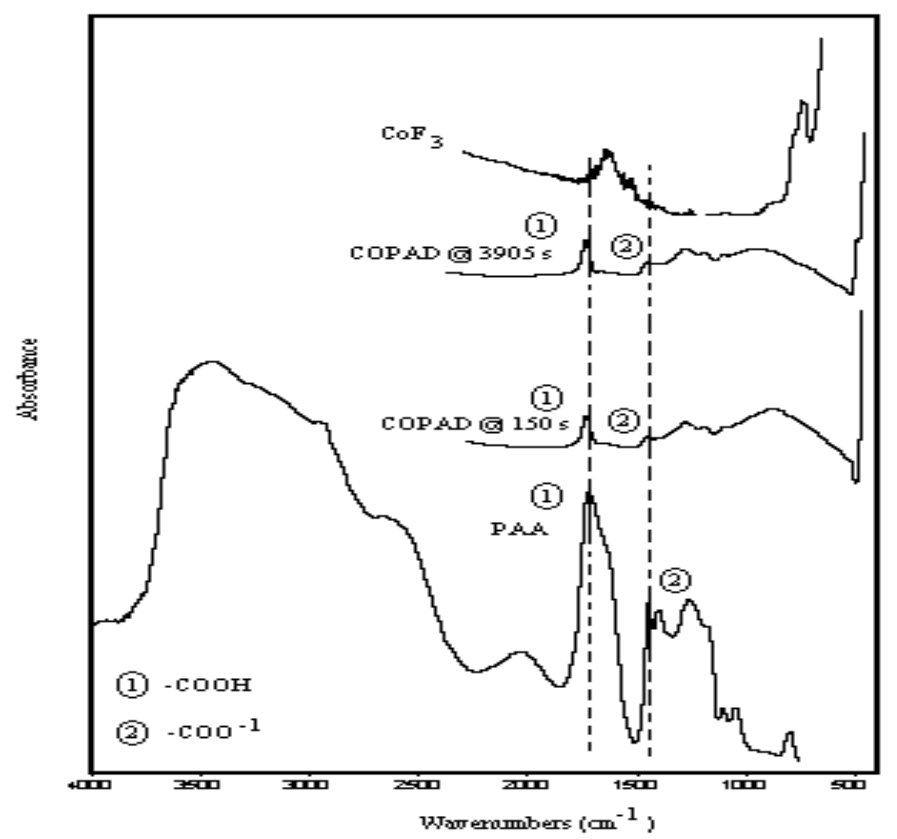

Fig. 5: Ftir Spectrum of Poly (Acrylic Acid) and Cobalt (Iii) Poly (Acrylate) Using $0.602 \sim \mathrm{Mol}$ of $\mathrm{Cof}_{3}$ and $0.0215 \sim \mathrm{Mol}$ of Paa or Paa/Cof $=$ 0.036 .

Figures 6, and 7 show the FTIR spectra of unreacted PAA and cobalt(III) poly(acrylate) salts with different molar ratios of PAA and $\mathrm{CoF}_{3}$. 


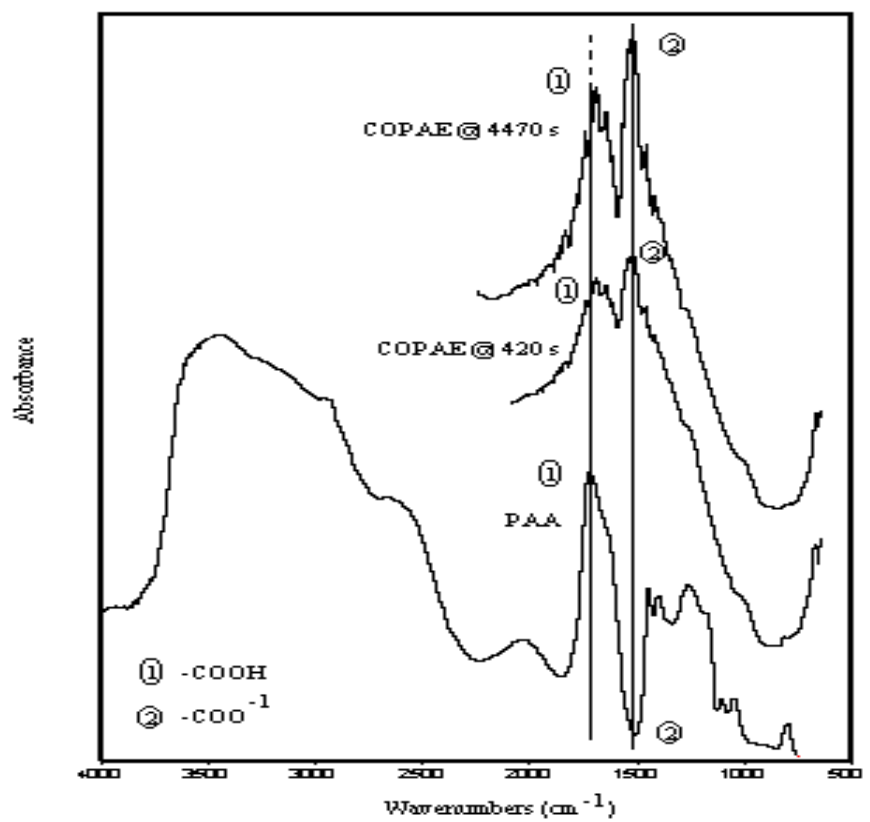

Fig. 6: Ftir Spectrum of Poly (Acrylic Acid) and Cobalt (Iii) Poly (Acrylate) Using $3.790 \sim \mathrm{Mol}$ of Cof and $0.0328 \sim \mathrm{Mol}$ of Paa or Paa/Cof ${ }_{3}=$ 0.009 .

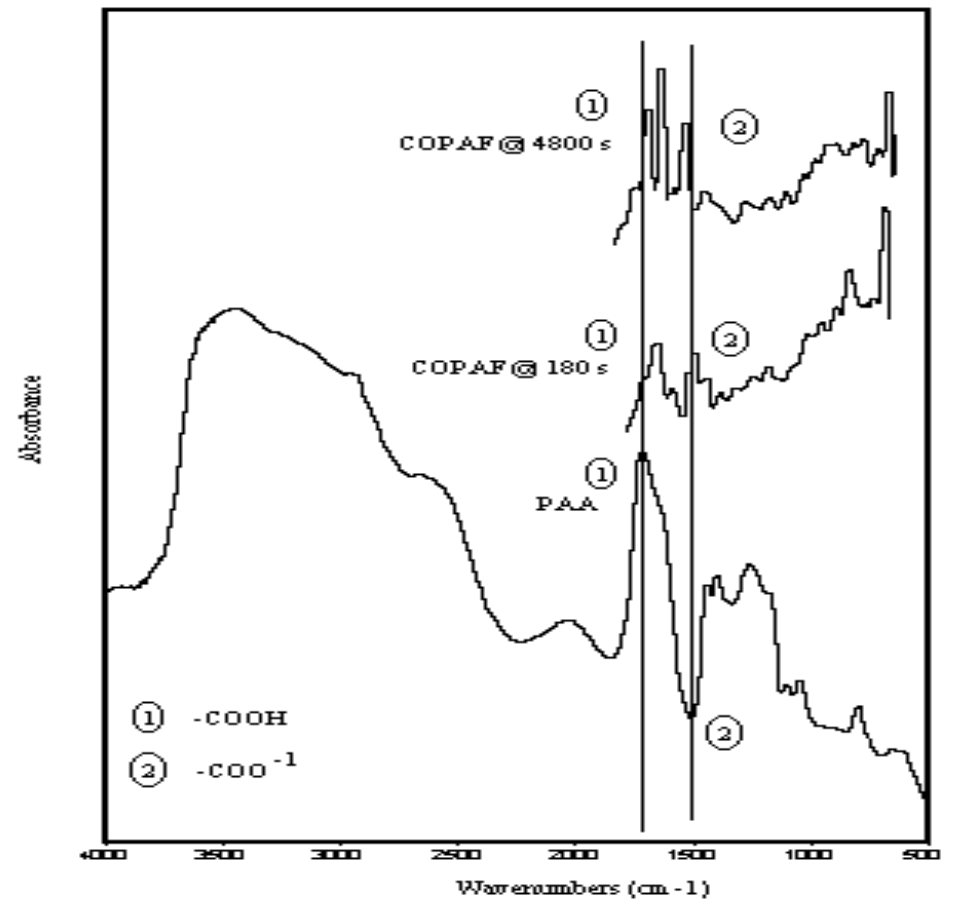

Fig. 7: Ftir Spectrum of Poly (Acrylic Acid) and Cobalt (Iii) Poly (Acrylate) Using $1.660 \sim \mathrm{Mol}$ of $\mathrm{Cof}_{3}$ and $0.0238 \sim \mathrm{Mol}$ of Paa or Paa/Cof $=$ 0.014 .

The general pattern of behaviour observed in the reacting pastes of PAA with $\mathrm{Co}$ (III) compounds was for the $\mathrm{C}=\mathrm{O}$ stetching band (at $1700 \mathrm{~cm}^{-1}$ ) of the carboxylic acid group to decline coincidently with the apparence and ehhancement of the $\mathrm{COO}^{-1}$ stretching bands of the metal carboxylates observed in the asymmetric stretch regions and the symmetric stretch regions. These results are indicative of an acid-base reaction.

The exact position of the asymmetrical and symmetrical $\mathrm{COO}^{-1}$ stretching modes can be used to obtain structural information. If the metal-carboxylate band is not ionic coordination complex are formed. There are bands with some covalent character then frequency shifts are to be expected. These shifts will depend on the metal-oxygen interaction and the nature of the coordination of the metal. Nakamoto [20] has suggested that there are four types of metal carboxylate structures: monodentate, bidentate bridging, bidentate chelating and ionic. It is possible to predict which structure is formed by knowing the wavenumber of the asymmetric and symmetric stretching vibrations. First, we 
define $\Delta v=v_{\text {as }}-v_{\mathrm{s}}$ where $v_{\text {as }}$ is the wavenumber of the asymmetric stretching and $v_{\mathrm{s}}$ is the wavenumber of the symmetric stretching.

When sodium poly(acrylate) is formed the carboxylate group is ionically bonded. The band of the carbonyl group at $\sim 1700 \mathrm{~cm}^{-1}$ is split in two new bands due to the asymmetric $\left(\sim 1500 \mathrm{~cm}^{-1}\right)$ and symmetric $\left(\sim 1400 \mathrm{~cm}^{-1}\right) \mathrm{stretching}^{-1}$ vibrations of the carboxylate anion formed. In this study, we found that the bands of the asymmetric and symmetric stretching to have frequencies of $1569 \mathrm{~cm}^{-1}$ and $1408 \mathrm{~cm}^{-1}$ respectively with a difference of $161 \mathrm{~cm}^{-1}$ [4].

All cobalt salts have $\Delta v$ between 105 and 200. Therefore, bidentate bridging structure may be tentatively assigned to cobalt(III) poly(acrylate). No matter which base is used $\left(\mathrm{Co}_{3} \mathrm{O}_{4}\right.$ or $\left.\mathrm{CoF}_{3}\right)$. However, there is a considerable proportion of unreacted PAA present in these reaction mixtures and free $\mathrm{OH}$ groups may play a role in the complex formation. Buckingham [6] stablishes the coordination number for cobalt(III) which is 6. This coordination number agrees with octahedrical stereochemistry structure. Therefore, octahedrical structure may be tentatively assigned to cobalt(III) poly(acrylate). Figure 8 shows schematically the proposed chemical structure of the hybrid formed [7], [10], [17], [18].

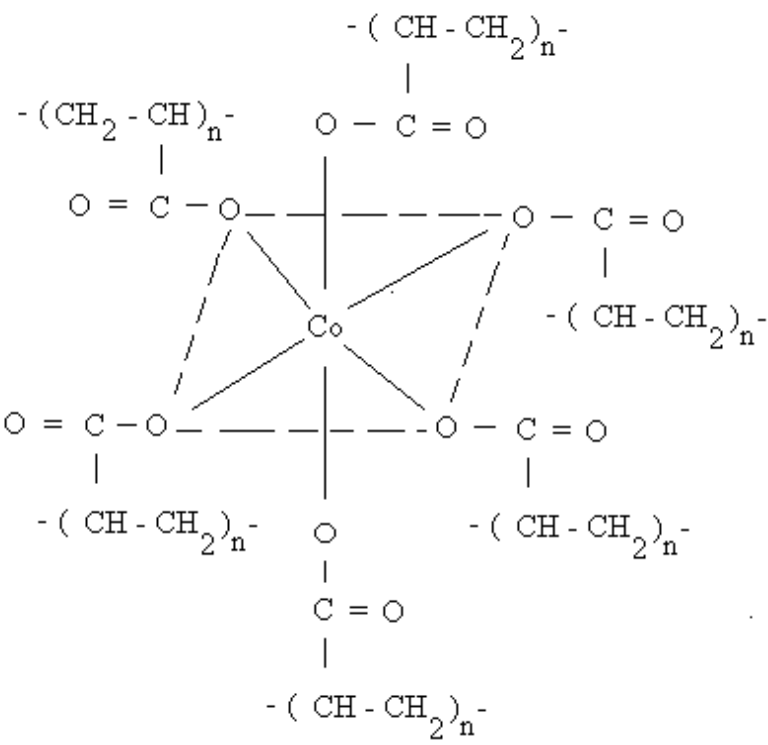

Fig. 8: Structure Propossed by Cobalt (Iii) Poly (Acrylate)

\section{Conclusions}

The oxide of the trivalent metal Co and the halide of the trivalent metal Co, react with aqueous solution of poly (acrylic acid) to form cobalt (III) poly (acrylate) a carboxylate salt. The halide of cobalt reacts with water of PAA first, then cobalt (III) hidroxide reacts with PAA to form the carboxylate salt. The structure could be a bidentate bridging. The coordination number for cobalt (III) is 6, and it agrees with octahedrical stereochemistry structure; therefore, octahedrical structure may be tentatively assigned to cobalt (III) poly (acrylate).

\section{References}

[1] A.D. Wilson, S. Crisp; "Organolithic Macromolecular Materials", Chapter 5, Applied Science Publishers, London, 1977.

[2] A. Padilla, J.L. Martinez, A. Sanchez, V.M. Casta-o; Mater. Lett, 12, 445 (1992) http://dx.doi.org/10.1016/0167-577X(92)90211-2.

[3] A. Wilson, J. Nicholson, "Acid-Base Cements", Cap. 5, Cambridge University Press, Cambridge U.K., 1993. http://dx.doi.org/10.1017/cbo9780511524813.

[4] B. Moody, "Comparative Inorganic Chemistry", 403-444, E. Arnold Division of Holder \& Stoughton, Londres, 1991.

[5] C. Alvarez-Gayosso, J. Palacios-Alquisira, V. Casta-o; Intern. J. Polymeric Mater., in press.

[6] D.A. Buckingham, "Structure and Stereochemistry of Coordination Compounds", Inorganic Biochemistry, Cap. 1, Vol. I, G.L. Eichhorn Editor, Elsevier Scientific Publishing Company, Amsterdam, 1973.

[7] F. Hoffmann, M. Cornelius, J. Morell, M. Froda, Angewandte Chemie 45, 3216 (2006). http://dx.doi.org/10.1002/anie.200503075.

[8] F. Oosawa, "Polyelectrolytes", Marcel Dekker, New York, 1971.

[9] G.B. Deacon, R.J. Phillips, Coordination Chemistry Reviews, 33, 227 (1980) http://dx.doi.org/10.1016/S0010-8545(00)80455-5.

[10] G. Hernández, F. Rojas, M. García, M. Canseco,V.M. Casta-o, Mater. Sci. \& Eng. A 355, 338 (2003) http://dx.doi.org/10.1016/S09215093(03)00101-1.

[11] H. Hu, J. Saniger, J. García-Alejandre, V. M. Casta-o, Mater. Lett. 12, 281 (1991) http://dx.doi.org/10.1016/0167-577X(91)90014-W.

[12] H. Hu, J. Saniger, V.M. Casta-o, Mater. Lett. 14, 83 (1992). http://dx.doi.org/10.1016/0167-577X(92)90179-N.

[13] H. Hu, J. Saniger, V.M. Casta-o, Mater. Lett. 16, 200 (1993). http://dx.doi.org/10.1016/0167-577X(93)90163-R.

[14] J. D. Lee, "Concise Inorganic Chemistry", Chapman \& Hall, 783-799, New York, 1991.

[15] J. M. Saniger, H. Hu, V. M. Casta-o, J. García-Alejandre, Characterization Techniques for the Solid Solution Interface, 169-176.

[16] J. Saniger, H. Hu, V. M. Casta-o, Mater. Lett. 15, 113 (1992) http://dx.doi.org/10.1016/0167-577X(92)90024-E. 
[17] J. Sun, S. Dong, E. Wang, J. Amer. Chem. Soc. 127, 13102 (2005) http://dx.doi.org/10.1021/ja0534809.

[18] J. Varela, V. Rodríguez, E. Rubio y V.M. Casta-o, J. New Mater. For Electrochem. Syst. 14, 59 (2011)

[19] J.W. Nicholson, S.J. Hawkins, E.A. Wasson; J. Mat. Sci., 4, 32 (1993)

[20] K: Nakamoto, "IR and RS of Inorganic and Coordination Compounds", Cap. 4, John Wiley \& Sons, New York, 1986.

[21] L. J. Bellamy "The Infra-red Spectra of Complex Molecules",Methuen, London, 1954.

[22] M.A. Moharram, N. Abdel-Hakeem, H. Shaheen, R.M. Ibrahim, J. Mater. Sci., 21, 1986, 1681-1698. http://dx.doi.org/10.1007/BF01114725.

[23] M. Nicho, J. Saniger, M. Ponce, A. Huanosta, V. Casta-o, J. Appl. Polym. Sci., 66:861 (1977). http://dx.doi.org/10.1002/(SICI)10974628(19971031)66:5<861::AID-APP6>3.0.CO;2-T.

[24] N.T. Woodberry; Tappi, 44, 1961, 156A-160A.

[25] R.G. Hill, S.A. Labok; J. Mat. Sci., 26, 67 (1991) http://dx.doi.org/10.1007/BF00576034.

[26] R. Nyquist, R.O. Kagel, "Infrared Spectra of Inorganic Compounds", Academic Press, New York, 1971. http://dx.doi.org/10.1016/b978-0-12523450-4.50005-5.

[27] R.P. Hopkins; Industrial \& Engineering Chemistry, 47, 1955, 2258-2265. http://dx.doi.org/10.1021/ie50551a024.

[28] S. Crisp, B.E. Kent, B.G. Lewis, A.J. Ferner, A.D. Wilson, J. Dent. Res., 59, (6), $1980,1055-1063$. http://dx.doi.org/10.1177/00220345800590060801.

[29] S. Crip, H.J. Prosser, A.D. Wilson, J. Mater. Sci., 11, 36 (1976). http://dx.doi.org/10.1007/BF00541072.

[30] S.M. Rabie, A. Sawaby, M.A. Moharram, A.M. Nassar, K.H. Tahon, J. Appl. Polym. Sci., 41, $445, \quad 1990$. http://dx.doi.org/10.1002/app.1990.070410301.

[31] T.W. Lambe, A.S. Michaels; Chemmical Engineering News, 32, 1954, 488-492. http://dx.doi.org/10.1021/cen-v032n006.p488.

[32] W. Ho-Shing, J. Heay-Chain, H. Jiann-Wen, J. Appl. Polym. Sci., 63, 1997, 89-101. http://dx.doi.org/10.1002/(SICI)10974628(19970103)63:1<89::AID-APP9>3.0.CO;2-O 